\title{
Heme oxygenase-1: a redoubtable response that limits reperfusion injury in the transplanted adipose liver
}

\author{
Karl A. Nath \\ Nephrology Research Unit, Mayo Clinic, 200 First Street, SW, 542 Guggenheim Building, Rochester, Minnesota 55905, USA. \\ Phone: (507) 284-1646; Fax: (507) 284-3757; E-mail: nath.karl@ mayo.edu.
}

Subjected to adversity, tissues often respond with redoubtable resourcefulness. Such resourcefulness enables tissues to endure and surmount circumstances inimical to their vitality. Salutary responses to such stresses are diverse and include processes that dissipate the brunt of the imposed insult, cytoprotective mechanisms that safeguard vulnerable cellular targets, metabolic adaptations that are energetically more favorable in the stressed state, reparative processes that restore the integrity of injured sites, regenerative responses that replace cells lost by necrosis or apoptosis, and, finally, remodeling that restores tissues to their original architecture.

In other instances, the outcome in the encounter between healthy tissues and unhealthy environments is one of widespread and irreparable tissue damage. Insults may overwhelm extant cellular defenses by their severity while, by their precipitancy of onset, insults may deny cells the opportunity to summon protective responses. Such cellular unpreparedness allows injurious biochemical processes to escalate unchecked and organelles to be critically disabled. However, the prior recruitment in these tissues of an appropriate protective response may permit them to withstand such insults that are otherwise overwhelming.

This capacity of tissues to resist perturbing influences not only applies to pathologic states but is also fundamental to physiologic ones. Homeostatic mechanisms exercised at systemic and local levels ensure optimal conditions for organ function in health and even in the face of a changing external environment. Depriving organs of their blood supply and their communication with the internal milieu, however, as occurs when donor organs are procured, initiates a series of cellular stresses that these harvested donor organs are ex-pected to successfully negotiate. Harvested organs are then maintained in the cold and in solutions that, despite improvements, remain an imperfect substitute for their natural environment. The resumption of their vascular supply restores much of what was deprived, but this reperfusion of organs subjected to varied amounts of warm and cold ischemia now ushers in a unique pathway of injury, namely, ischemia/reperfusion (I/R) damage. This pathway may impair vascular and hemodynamic integrity, inflict acute sublethal or lethal cell injury, and instigate an acute inflammatory response (1). I/R injury may induce primary nonfunction of the graft in the immediate posttransplant period, and such injury, in turn, predisposes organs to acute rejection and chronic allograft dysfunction. Whereas the biochemical basis for I/R injury is complex, at least a component of such injury resides in enhanced generation of reactive oxygen species.

Substrates such as fat can fuel oxidative stress incurred during reperfusion, and the presence of large amounts of lipid in donor organs, as found not infrequently in harvested livers, may render such organs prone to nonfunction in the early posttransplant phase. Amersi et al. in this issue reasoned that the susceptibility of such larded livers to oxidation and to other mechanisms of injury during reperfusion may be abrogated by the prior recruitment of a cytoprotective, antioxidant response; they focused, presciently, on one such response, heme oxygenase-1 (HO-1) (2). Using the obese Zucker rat, these investigators demonstrated that prior induction of HO-1 markedly improved hepat- ic function in an ex vivo perfusion model and strikingly reduced not only hepatic injury in syngeneic hepatic transplants but also attendant mortality in the recipients of these grafts.

$\mathrm{HO}$ is the rate-limiting enzyme in heme degradation $(3,4)$ (Figure 1). HO facilitates the opening of the heme ring and its conversion to biliverdin, in the course of which carbon monoxide evolves and iron is released; iron, in turn, fosters the synthesis of ferritin, whereas biliverdin is subsequently converted to bilirubin. $\mathrm{HO}$ was discovered in the course of the inquiry into the capacity of the reticuloendothelial system to degrade aged hemoglobin; for much of the time since its discovery, $\mathrm{HO}$ was studied within the confines of heme metabolism. However, interest in $\mathrm{HO}$ rapidly grew when it was recognized that one of its isozymes, HO-1, was readily, and often copiously, induced by diverse insults including heme, oxidants, ischemia, hyperoxia, hypoxia, and cytokines $(3,4)$. Not only was the enzyme induced, but, in an accumulating number of circumstances, such induction conferred protection against tissue injury (3-8).

The basis for the protective effects of HO- 1 in the present and most of the previous studies remains very much a matter of conjecture. Such conjecture draws upon the well-established, proximate biochemical actions of $\mathrm{HO}$ as the basis for this cytoprotection (Figure 1), including the reduction in cellular levels of a potential toxicant (heme) and the procurement of potentially cytoprotective species (ferritin, carbon monoxide, biliverdin/biliru- 
bin). The heme prosthetic group is ubiquitously disposed within cells, contained as it is in proteins that carry or store oxygen, mitochondrial and microsomal cyto-chromes, and numerous other proteins. Disengaged from its linkage with such proteins - as when cells are injured - heme provides a readily transmissible pro-oxidant species that can destabilize cellular organelles; induced $\mathrm{HO}-1$ may restrain the buildup of heme that occurs in injured cells $(3,4)$. Ferritin may confer cytoprotection by its iron-sequestering capability and, possibly, other effects (4). Carbon monoxide is an antiapoptotic, anti-inflammatory, and vasodilatory molecule $(3,4)$; bili-verdin and bilirubin are antioxidant, anti-inflammatory species $(3,4,9)$. Such actions, arising from induced $\mathrm{HO}-1$, would be beneficial to cells under stress. However, there are several caveats (4). Few, if any, studies have directly demonstrated that any of these products of HO-1 specifically account for the cytoprotection conferred by in-duced HO-1. Ironically, at one time or another in their history, each of these products of $\mathrm{HO}$ has been viewed as a perpetrator of, rather than a protector against, tissue injury. Additionally, in certain injured states, induced $\mathrm{HO}-1$ seems an uninvolved bystander rather than a protectant against such injury. Finally, there is a sense that the beneficial effects of HO- 1 in injured tissue may involve some recondite mechanism that transcends any one of the currently invoked pathways.

The unique and remarkable contribution of the study of Amersi et al. is that it speaks to a current clinical exigency the relative lack of cadaveric donor organs. That suboptimal donor livers can be salvaged and conditioned to be used effectively in transplantation provides a clinically relevant strategy for the augmentation of the donor pool. This strategy is applicable to other organs besides the liver. For example, kidneys from older donors are increasingly employed in transplantation; such kidneys may exhibit varying amounts of sclerosis and are prone to dysfunction in the posttransplant period. An approach similar to the one employed by Amersi et al. may enhance the performance of these older kidneys. The broader significance of the present study, however, goes beyond such clinical applications, extending as it does to an overarching biologic issue: lifespan and its determinants. The longevity of various species correlates, broadly and remarkably, with the ca-pacity of respective cells to withstand and respond to oxidant and other forms of stress (10). This capacity is not fixed; rather, as shown by Amersi et al. (2), resistance to stress can be enhanced by directly delivering or pharmacologically activating a particular gene. In this regard, and in the described setting, induced HO- 1 is dramatically protective: HO-1 prolongs the longevity of the graft and the survival of the recipient, even in the face of adversity.

1. Halliwell, B., and Gutteridge, J.M.C. 1999. Free radicals in biology and medicine. Oxford University Press. Oxford, United Kingdom. 617-783.

2. Amersi, F., et al. 1999. Upregulation of heme oxygenase-1 protects genetically fat Zucker rat livers from ischemia/reperfusion injury. J. Clin. Invest. 104:1631-1639

3. Maines, M.D. 1997. The heme oxygenase system: a regulator of second messenger gases. Annu. Rev. Pharmacol. Toxicol. 37:517-554.

4. Platt,J.L., and Nath, K.A. 1998. Heme oxygenase: protective gene or Trojan horse. Nat. Med. 4:1364-1365.

5. Soares, M.P, et al. 1998. Expression of heme oxygenase-1 can determine cardiac xenograft survival. Nat. Med. 4:1073-1077.

6. Hancock, W.W., Buelow, R., Sayegh, M.H., and Turka, L.A. 1998. Antibody-induced transplant arteriosclerosis is prevented by graft expression of anti-oxidant and anti-apoptotic genes. Nat. Med. 4:1392-1396.

7. Yet, S.-F., et al. 1999. Hypoxia induces severe right ventricular dilatation and infarction in heme oxygenase-1 null mice. J. Clin. Invest. 103:R23-R29.

8. Otterbein, L.E., et al. 1999. Exogenous administration of heme oxygenase- 1 by gene transfer provide protection against hyperoxia-induced lung injury. J. Clin. Invest. 103:1047-1054.

9. Dore, S., et al. 1999. Bilirubin, formed by activation of heme oxygenase-2, protects neurons against oxidative stress injury. Proc. Natl. Acad. Sci. USA. 96:2445-2450.

10. Kapahi, P., Boulton, M.E., and Kirkwood, T.B.L. 1999. Positive correlation between mammalian lifespan and cellular resistance to stress. Free Radic. Biol. Med. 26:495-500. 